\title{
Bilayer crystals of charged magnetic dipoles: Structure and phonon spectrum
}

\author{
I. R. O. Ramos, ${ }^{1}$ W. P. Ferreira,,${ }^{1, *}$ F. F. Munarin, ${ }^{2}$ G. A. Farias, ${ }^{1}$ and F. M. Peeters ${ }^{1,3}$ \\ ${ }^{1}$ Departamento de Física, Universidade Federal do Ceará, Caixa Postal 6030, Campus do Pici, 60455-760 Fortaleza, Ceará, Brazil \\ ${ }^{2}$ Centro de Tecnologia, Bloco 710, Universidade Federal do Ceará, Campus do Pici, 60455-760 Fortaleza, Ceará, Brazil \\ ${ }^{3}$ Department of Physics, University of Antwerp, Groenenborgerlaan 171, B-2020 Antwerpen, Belgium
}

(Received 13 February 2012; published 24 May 2012)

\begin{abstract}
We study the structure and phonon spectrum of a two-dimensional bilayer system of classical charged dipoles oriented perpendicular to the plane of the layers for equal density in each layer. This system can be tuned through six different crystalline phases by changing the interlayer separation or the charge and/or dipole moment of the particle. The presence of the charge on the dipole particles is responsible for the nucleation of five staggered phases and a disordered phase which are not found in the magnetic dipole bilayer system. These extra phases are a consequence of the competition between the repulsive Coulomb and the attractive dipole interlayer interaction. We present the phase diagram and determine the order of the phase transitions. The phonon spectrum of the system was calculated within the harmonic approximation, and a nonmonotonic behavior of the phonon spectrum is found as a function of the effective strength of the interparticle interaction. The stability of the different phases is determined.
\end{abstract}

DOI: 10.1103/PhysRevE.85.051404

PACS number(s): 64.70.pv, 63.20.D-, 63.22.Np, 64.70.Nd

\section{INTRODUCTION}

Strongly repulsive interacting particles crystallize for a certain range of density and temperature. This has been found in systems of rather different nature and therefore the study of the structural and dynamical properties of such a crystalline phase is of fundamental interest. The crystallization phenomenon of strongly interacting particles was originally predicted for an electron gas [Wigner crystal (WC)] by E. P. Wigner in 1934 [1]. Up to now, the original three-dimensional (3D) WC of electrons is not yet observed experimentally mainly due to defects and imperfections in real lattice structures. But experimental evidence of the WC was found in 1979 in a two-dimensional (2D) system of electrons on the surface of liquid helium [2]. Nowadays, the term Wigner crystal is used in a broad sense for the crystalline state of clusters of strongly interacting particles. Such Wigner crystallization has also been observed in atomic and molecular clusters [3-5] and in several nonelectronic classical systems as colloids [6-11], complex dusty plasma [12], and metallic spheric balls [13].

For the particular case of classical systems (e.g., charged or magnetic colloidal particles [14]), crystallization is observed if the interaction potential energy overcomes the kinetic energy of the particles and correlation effects dominate the long-range structure of the system [15]. More specifically, the thermodynamic state of the system is characterized by the coupling parameter $\Gamma$, defined as the average of the ratio between the interaction potential energy and the kinetic energy. For a 2D classical system of charged particles with Coulomb interaction $\Gamma=q^{2} \sqrt{\pi n} / k_{B} T$, where $q$ is the charge of each particle, $n$ the density, $k_{B}$ the Boltzmann constant, and $T$ the temperature. For $\Gamma<1$, the kinetic energy largely dominates the interacting term and the system behaves as a classical gas. For intermediate values $1 \leqslant \Gamma \leqslant 100$, particles become more correlated and a liquid state is found. For $\Gamma>100$, the interacting potential energy dominates the kinetic energy,

*wandemberg@fisica.ufc.br particles become strongly correlated and the system typically transits to a crystalline phase for $\Gamma \sim 130$.

In a $2 \mathrm{D}$ system of purely repulsive interacting particles the ground-state configuration is found to be the hexagonal lattice $[16,17]$. However, a more interesting scenario is observed if a $2 \mathrm{D}$ system of particles with pure repulsive interaction is arranged in a bilayer structure. In this case, the set of possible ground-state configurations is richer, and many other 2D structures, not observed in the single-layer case, now appear as the minimum energy configuration. Goldoni and Peeters [18] showed that the hexagonal lattice is the ground state only when the separation between layers is zero or larger than a critical value. In the latter case, the hexagonal lattice in each layer are displaced with respect to each other (staggered hexagonal phase). For intermediate distances between the layers, staggered square, rectangular, and rhombic phases become the ground state.

In a 2D system of magnetic dipoles oriented perpendicularly to the layers, Lu et al. [19] showed that, independently of the distance between the layers, the hexagonal phase is the minimum energy structure in each layer, and the dipoles in the different layers are aligned along the direction perpendicular to the layers (matching hexagonal phase). In addition, a reentrant melting temperature, which was related to the anisotropic nature of the dipole interaction, was predicted in this case. Magnetic 2D systems of colloidal particles appear yet in many interesting recent studies [9,10,20-22].

Motivated by modern technical methods of synthesizing particles and the assembly of colloidal particles in controlled structures [23] we study in this paper a 2D classical bilayer system of charged magnetic dipoles directed perpendicular to the layers (which can be realized by the application of a magnetic field). Such particles have recently been produced using magnetic colloidal particles [7] with electric stabilization [24]. Note that in an electric stabilized colloidal system the charge of the colloids can in principle be controlled by the $\mathrm{PH}$ of the solution by adding or removing salt to or from the solvent [25]. Furthermore the magnetic moment of the paramagnetic particles is tunable by the strength of the external 
magnetic field. In a single layer both the Coulomb and the magnetic interaction lead to a repulsion between the particles favoring the formation of a 2D Wigner lattice. Between the layers the particles exert a repulsive Coulomb interaction while the magnetic interaction is attractive. Depending on the relative strength between the magnetic and Coulomb interaction the particles in both layers tend to be staggered or on top of each other. In the present paper, we study the ground-state configurations and the frequencies of the phonon modes as a function of the separation between the layers and a parameter which is related to the ratio between the dipole moment $(\mu)$ and the charge $(Q)$ of the particles $\left(\lambda=\mu^{2} n / Q^{2}\right.$, with $n$ the density of particles).

Our paper is organized as follows. In Sec. II we introduce the model, define the important parameters used to characterize the system and calculate the total energy of the system. In Sec. III the results for the ground-state configurations are presented and discussed as a function of the separation between the layers and $\lambda$. In Sec. IV we present the methodology used to calculate the phonon spectrum and discuss the numerical results. Our conclusions are given in Sec. V.

\section{MODEL}

We study a two-dimensional classical crystal of charged dipole particles with total density $n$ arranged in a bilayer structure. The particles are evenly distributed over the layers ( $x y$ plane), which are separated by a distance $d$ along the $z$ axis. Each particle has charge $Q$ and magnetic dipole moment $\vec{\mu}=\mu \hat{e}_{z}$ oriented perpendicular to the layers. Thus, the interparticle interaction consists of a repulsive Coulomb term $Q^{2} /\left|\vec{r}_{1}-\vec{r}_{2}\right|$ and a dipole interaction term $\mu^{2} /\left|\vec{r}_{1}-\vec{r}_{2}\right|^{3}$. For convenience we included the dielectric constant $\epsilon$ of the medium into $Q^{2}$ and therefore, $Q / \sqrt{\epsilon}$ is the real charge of the particles.

In order to confine the colloidal particles in each layer into a plane one can make use of, for example, glass plates. Because of the difference between the dielectric constants of the glass plates and the water environment where the colloids are moving in, this will lead to image charges as were, for example, discussed by Peeters and $\mathrm{Wu}$ [26]. But because the dielectric constant of water $(\epsilon=80)$ is much larger than of the confining glass plates the induced image charges have the same charge as the colloidal particles. This will have two effects: (1) The colloidal particles will be repelled by the glass plates and will therefore form a 2D layer in the middle between the two glass plates, and (2) the intercolloid repulsive interaction will increase which can, to some extent, be modeled by replacing the charge $Q$ by an effective charge $Q^{*}>Q$. Therefore, including this dielectric mismatch effect will not qualitatively modify our results.

Typically we consider colloidal particles containing magnetic ions exhibiting paramagnetic behavior and thus a magnetic field is applied in the $z$ direction aligning all magnetic moments in the $z$ direction. The considered crystal structures are 2D lattices in which the unit cell consists of two particles, one in each layer, where we will label the lattices in different layers by $\mathrm{A}$ and $\mathrm{B}$. The equilibrium positions of the particles in each layer are given by $\vec{R}_{A}=l_{1} \vec{a}_{1}+l_{2} \vec{a}_{2}$, and $\vec{R}_{B}=l_{1} \vec{a}_{1}+l_{2} \vec{a}_{2}+\vec{c}$, where $l_{1}$ and $l_{2}$ are integers, $\vec{a}_{1}$ and $\vec{a}_{2}$ are the primitive vectors; $\vec{c}$ is a two-dimensional vector which describes the shift of lattice $\mathrm{B}$ with respect to $\mathrm{A}$ in the $x y$ plane. For $\vec{c}=0$ the lattices are not displaced, and are exactly on top of each other (matched case). The case $\vec{c} \neq 0$ implies staggered lattices. Because of equal density of particles in both layers, the lattice structure in both layers is the same.

The total interaction energy is given by

$$
E^{t}=E_{e l}^{t}+E_{\mathrm{mag}}^{t}
$$

with the Coulomb interaction energy,

$$
\begin{aligned}
E_{e l}^{t}= & \frac{1}{2} \sum_{R_{A} \neq R_{A}^{\prime}} \frac{Q^{2}}{\left|\vec{R}_{A}-\vec{R}_{A}^{\prime}\right|}+\frac{1}{2} \sum_{R_{B} \neq R_{B}^{\prime}} \frac{Q^{2}}{\left|\vec{R}_{B}-\vec{R}_{B}^{\prime}\right|} \\
& +\sum_{R_{A}, R_{B}} \frac{Q^{2}}{\sqrt{\left|\vec{R}_{A}-\vec{R}_{B}\right|^{2}+d^{2}}}
\end{aligned}
$$

where $d$ is the separation between the layers. The dipole-dipole interaction energy is

$$
\begin{aligned}
E_{\text {mag }}^{t}= & \frac{1}{2} \sum_{R_{A} \neq R_{A}^{\prime}} \frac{\mu^{2}}{\left|\vec{R}_{A}-\vec{R}_{A}^{\prime}\right|^{3}}+\frac{1}{2} \sum_{R_{B} \neq R_{B}^{\prime}} \frac{\mu^{2}}{\left|\vec{R}_{B}-\vec{R}_{B}^{\prime}\right|^{3}} \\
& +\sum_{R_{A}, R_{B}} \frac{\mu^{2}\left(\left|\vec{R}_{A}-\vec{R}_{B}\right|^{2}-2 d^{2}\right)}{\left[\left|\vec{R}_{A}-\vec{R}_{B}\right|^{2}+d^{2}\right]^{\frac{5}{2}}} .
\end{aligned}
$$

Since the layers are equivalent, it is convenient to write the total energy per particle $E$ as

$$
E=\frac{E^{t}}{N}=E_{e l}+E_{\mathrm{mag}}
$$

where the total Coulomb $\left(E_{e l}\right)$ and magnetic $\left(E_{\text {mag }}\right)$ energy per particle can be split as

$$
\begin{aligned}
E_{e l} & =\frac{1}{2}\left(E_{0 E}+E_{I E}\right), \\
E_{\mathrm{mag}} & =\frac{1}{2}\left(E_{0 M}+E_{I M}\right),
\end{aligned}
$$

where

$$
\begin{aligned}
& E_{0 E}=\sum_{\vec{R} \neq \overrightarrow{0}} \frac{Q^{2}}{|\vec{R}|}, \\
& E_{0 M}=\sum_{\vec{R} \neq \overrightarrow{0}} \frac{\mu^{2}}{|\vec{R}|^{3}},
\end{aligned}
$$

are the Coulomb and magnetic interaction energy per particle in each layer, respectively, and $\vec{R}=l_{1} \vec{a}_{1}+l_{2} \vec{a}_{2}$. On the other hand,

$$
\begin{aligned}
& E_{I E}=\sum_{\vec{R}} \frac{Q^{2}}{\left(|\vec{R}+\vec{c}|^{2}+d^{2}\right)^{1 / 2}}, \\
& E_{I M}=\sum_{\vec{R}} \frac{\mu^{2}\left(|\vec{R}+\vec{c}|^{2}-2 d^{2}\right)}{\left(|\vec{R}+\vec{c}|^{2}+d^{2}\right)^{5 / 2}},
\end{aligned}
$$

are the Coulomb and magnetic interaction energy per particle between particles in distinct layers, respectively. Following the procedure developed in Refs. [16-19] we define now the 
auxiliary functions:

$$
\begin{aligned}
& T_{0}(\vec{r}, \vec{q})=e^{-i \vec{q} \cdot \vec{r}} \sum_{\vec{R}} \frac{e^{i \vec{q} \cdot(\vec{r}-\vec{R})}}{|\vec{r}-\vec{R}|}-\frac{1}{r} \\
& T_{I}(\vec{r}, \vec{q})=e^{-i \vec{q} \cdot \vec{r}} \sum_{\vec{R}} \frac{e^{i \vec{q} \cdot(\vec{r}-\vec{R}+\vec{c})}}{\left[|\vec{r}-\vec{R}+\vec{c}|^{2}+d^{2}\right]^{1 / 2}}, \\
& \psi_{0}(\vec{r}, \vec{q})=e^{i \vec{q} \cdot \vec{r}} \sum_{\vec{R} \neq \overrightarrow{0}} \frac{e^{-i \vec{q} \cdot(\vec{r}+\vec{R})}}{|\vec{r}+\vec{R}|^{3}}, \\
& \psi_{I}(\vec{r}, \vec{q})=e^{i \vec{q} \cdot \vec{r}} \sum_{\vec{R}}\left(\frac{e^{-i \vec{q} \cdot(\vec{r}+\vec{R}+\vec{c})}}{|\vec{r}+\vec{R}+\vec{c}|^{3}}+\frac{-3 d^{2} e^{-i \vec{q} \cdot(\vec{r}+\vec{R}+\vec{c})}}{|\vec{r}+\vec{R}+\vec{c}|^{5}}\right) .
\end{aligned}
$$

The function $\psi_{I}(\vec{r}, \vec{q})$ can also be written as

$$
\psi_{I}(\vec{r}, \vec{q})=\psi_{I 1}(\vec{r}, \vec{q})-3 d^{2} \psi_{I 2}(\vec{r}, \vec{q}),
$$

with

$$
\begin{aligned}
& \psi_{I 1}(\vec{r}, \vec{q})=\sum_{\vec{R}} \frac{e^{-i \vec{q} \cdot(\vec{R}+\vec{c})}}{|\vec{r}+\vec{R}+\vec{c}|^{3}}, \\
& \psi_{I 2}(\vec{r}, \vec{q})=\sum_{\vec{R}} \frac{e^{-i \vec{q} \cdot(\vec{R}+\vec{c})}}{|\vec{r}+\vec{R}+\vec{c}|^{5}},
\end{aligned}
$$

where $|\vec{r}+\vec{R}+\vec{c}| \equiv\left(|\vec{r}+\vec{R}+\vec{c}|^{2}+d^{2}\right)^{1 / 2}$. Using Eqs. (8)(10) we can write Eqs. (6) and (7) as

$$
\begin{aligned}
& E_{0 E}=Q^{2} \lim _{\vec{r} \rightarrow 0} T_{0}(\vec{r}, \overrightarrow{0}), \\
& E_{I E}=Q^{2} \lim _{\vec{r} \rightarrow 0} T_{I}(\vec{r}, \overrightarrow{0}), \\
& E_{0 M}=\mu^{2} \lim _{\vec{r} \rightarrow 0} \psi_{0}(\vec{r}, \overrightarrow{0}), \\
& E_{I M}=\mu^{2} \lim _{\vec{r} \rightarrow 0} \psi_{I}(\vec{r}, \overrightarrow{0}) .
\end{aligned}
$$

Due to the long-range nature of the interactions, we use the Ewald summation method in order to improve the convergence of the energy expressions. Therefore, for the Coulomb interaction, Eqs. (8a) and (8b) are rewritten as [16-18]

$$
\begin{aligned}
T_{0}(\vec{r}, \vec{q})= & \sqrt{n / 2} \sum_{\vec{G}} e^{-i(\vec{q}+\vec{G}) \cdot \vec{r}} \Phi\left(\frac{|\vec{q}+\vec{G}|^{2}}{2 \pi n}\right) \\
& +\sqrt{n / 2} \sum_{\vec{R} \neq \overrightarrow{0}} e^{-i \vec{q} \cdot \vec{R}} \Phi\left(\pi n|\vec{r}-\vec{R}|^{2} / 2\right) \\
& +\sqrt{n / 2} \Phi\left(\pi n|\vec{r}|^{2} / 2\right)-\frac{1}{r},
\end{aligned}
$$

$$
\begin{aligned}
T_{I}(\vec{r}, \vec{q})= & \sqrt{n / 2} \sum_{\vec{G}} e^{-i(\vec{q}+\vec{G}) \cdot \vec{r}} e^{-i \vec{G} \cdot \vec{c}} \Psi\left(\frac{|\vec{q}+\vec{G}|^{2}}{2 \pi n}, \pi \eta^{2}\right) \\
& +\sqrt{n / 2} \sum_{\vec{R}} e^{-i \vec{q} \cdot(\vec{R}-\vec{c})} \Phi\left(\pi\left[n|\vec{r}-\vec{R}+\vec{c}|^{2} / 2+\eta^{2}\right]\right),
\end{aligned}
$$

where $G$ are arbitrary reciprocal lattice vectors given by $\vec{G}=l_{1} \vec{b}_{1}+l_{2} \vec{b}_{2}\left(l_{1}, l_{2}\right.$ are integers $)$ and $\vec{b}_{1}, \vec{b}_{2}$ are the primitive translation vectors of the reciprocal lattice. The functions,

$$
\Phi(x)=\sqrt{\frac{\pi}{x}} \operatorname{erfc}(\sqrt{x}),
$$

and

$$
\begin{aligned}
\Psi(x, y)= & \frac{1}{2} \sqrt{\frac{\pi}{x}}\left[e^{\sqrt{4 x y}} \operatorname{erfc}(\sqrt{x}+\sqrt{y})\right. \\
& \left.+e^{-\sqrt{4 x y}} \operatorname{erfc}(\sqrt{x}-\sqrt{y})\right]
\end{aligned}
$$

rapidly converge to zero for large values of their arguments. $\operatorname{erfc}(x)$ is the complementary error function, and $\eta=d \sqrt{n / 2}$ is a dimensionless parameter proportional to the separation between the two layers. By considering Eqs. (13) and (14), Eqs. (11a) and (11b) can be written as

$$
E_{0 E}=Q^{2} \sqrt{n / 2} A
$$

where

$$
A=2 \sum_{\vec{R} \neq \overrightarrow{0}} \Phi\left(\pi n|\vec{R}|^{2} / 2\right)-4,
$$

and

$$
E_{I E}=Q^{2} \sqrt{n / 2} B(\eta)
$$

where,

$$
\begin{aligned}
B(\eta)= & \sum_{\vec{R}} \Phi\left(\pi\left[n|\vec{R}+\vec{c}|^{2} / 2+\eta^{2}\right]\right) \\
& +\sum_{\vec{G} \neq \overrightarrow{0}} e^{-i \vec{G} \cdot \vec{c}} \Psi\left(\frac{|\vec{q}+\vec{G}|^{2}}{2 \pi n}, \pi \eta^{2}\right) \\
& +2\left\{\pi \eta \times \operatorname{erfc}(\sqrt{\pi} \eta)-e^{-\pi \eta^{2}}\right\} .
\end{aligned}
$$

A similar approach is considered for the magnetic interaction. In this case, following Ref. [19], rapidly convergent expressions are obtained:

$$
\begin{aligned}
\psi_{0}(\vec{r}, \vec{q})= & \frac{\pi n}{2} \sum_{\vec{G}} e^{i(\vec{q}+\vec{G}) \cdot \vec{r}}\left[\frac{4 \varepsilon}{\sqrt{\pi}} e^{-|\vec{q}+\vec{G}|^{2} / 4 \varepsilon^{2}}-2|\vec{q}+\vec{G}| \operatorname{erfc}\left(\frac{|\vec{q}+\vec{G}|}{2 \varepsilon}\right)\right]+\left[\frac{2 \varepsilon e^{-\varepsilon^{2} r^{2}}}{\sqrt{\pi} r^{2}}-\frac{\operatorname{erf}(\varepsilon r)}{r^{3}}\right] \\
& +\sum_{\vec{R} \neq \overrightarrow{0}} e^{-i \vec{q} \cdot \vec{R}}\left[\frac{\operatorname{erfc}(\varepsilon|\vec{R}+\vec{r}|)}{|\vec{R}+\vec{r}|^{3}}+\left(\frac{2 \varepsilon}{\sqrt{\pi}}\right) \frac{e^{-\varepsilon^{2}|\vec{R}+\vec{r}|^{2}}}{|\vec{R}+\vec{r}|^{2}}\right]
\end{aligned}
$$




$$
\begin{aligned}
\psi_{I}(\vec{r}, \vec{q})= & \frac{\pi n}{2} \sum_{\vec{G}} e^{i(\vec{q}+\vec{G}) \cdot \vec{r}} e^{i \vec{G} \cdot \vec{c}}\left[\frac{4 \varepsilon}{\sqrt{\pi}} e^{-\frac{|\vec{q}+\vec{G}|^{2}}{4 \varepsilon^{2}}-\varepsilon^{2} d^{2}}-e^{-|\vec{q}+\vec{G}| d}|\vec{q}+\vec{G}| \operatorname{erfc}\left(\frac{|\vec{q}+\vec{G}|}{2 \varepsilon}-\varepsilon d\right)\right. \\
& \left.-e^{|\vec{q}+\vec{G}| d}|\vec{q}+\vec{G}| \operatorname{erfc}\left(\frac{|\vec{q}+\vec{G}|}{2 \varepsilon}+\varepsilon d\right)\right]+\sum_{\vec{R}} e^{-i \vec{q} \cdot(\vec{R}+\vec{c})}\left[\frac{\operatorname{erfc}(\varepsilon|\vec{R}+\vec{c}+\vec{r}|)}{|\vec{R}+\vec{c}+\vec{r}|^{3}}+\left(\frac{2 \varepsilon}{\sqrt{\pi}}\right) \frac{e^{-\varepsilon^{2}|\vec{R}+\vec{c}+\vec{r}|^{2}}}{|\vec{R}+\vec{c}+\vec{r}|^{2}}\right. \\
& \left.-3 d^{2}\left(\frac{\operatorname{erfc}(\varepsilon|\vec{R}+\vec{c}+\vec{r}|)}{|\vec{R}+\vec{c}+\vec{r}|^{5}}+\left(\frac{2 \varepsilon}{3 \sqrt{\pi}}\right) \frac{\left(3+2 \varepsilon^{2}|\vec{R}+\vec{c}+\vec{r}|^{2}\right) e^{-\varepsilon^{2}|\vec{R}+\vec{c}+\vec{r}|^{2}}}{|\vec{R}+\vec{c}+\vec{r}|^{4}}\right)\right],
\end{aligned}
$$

where $|\vec{R}+\vec{c}+\vec{r}| \equiv\left(|\vec{R}+\vec{c}+\vec{r}|^{2}+d^{2}\right)^{1 / 2}$, and the parameter $\varepsilon>0$ is related to the inverse of the average distance between particles in the same layer (i.e., $\varepsilon=1 / r_{0}=\sqrt{\pi n / 2}$ ). In this case, Eqs. (11c) and (11d) can be written, respectively, as

$$
E_{0 M}=\mu^{2}(n / 2)^{3 / 2} C,
$$

where

$$
\begin{aligned}
C= & \sum_{\vec{G}}\left[4 \pi e^{-|\vec{G}|^{2} / 2 \pi n}-\frac{2|\vec{G}| \pi}{\sqrt{n / 2}} \operatorname{erfc}\left(\frac{|\vec{G}|}{2 \sqrt{\pi n / 2}}\right)\right] \\
& +\sum_{\vec{R} \neq \overrightarrow{0}}\left[\frac{\operatorname{erfc}(\sqrt{\pi n / 2}|\vec{R}|)}{(n / 2)^{3 / 2}|\vec{R}|^{3}}+\left(\frac{4}{n}\right) \frac{e^{-\pi n|\vec{R}|^{2} / 2}}{|\vec{R}|^{2}}\right]-\frac{4 \pi}{3},
\end{aligned}
$$

and

$$
E_{I M}=\mu^{2}(n / 2)^{3 / 2} D(\eta)
$$

where

$$
\begin{aligned}
D(\eta)= & \sum_{\vec{G}} e^{i \vec{G} \cdot \vec{c}}\left[4 \pi e^{-\frac{|\vec{G}|^{2}}{2 \pi n}-\pi \eta^{2}}\right. \\
& -\frac{\pi|\vec{G}|}{\sqrt{n / 2}} e^{-|\vec{G}| \eta / \sqrt{n / 2}} \operatorname{erfc}\left(\frac{|\vec{G}|}{2 \sqrt{\pi n / 2}}-\sqrt{\pi} \eta\right) \\
& \left.-\frac{\pi|\vec{G}|}{\sqrt{n / 2}} e^{|\vec{G}| \eta / \sqrt{n / 2}} \operatorname{erfc}\left(\frac{|\vec{G}|}{2 \sqrt{\pi n / 2}}+\sqrt{\pi} \eta\right)\right] \\
& +\sum_{\vec{R}}\left[\frac{\operatorname{erfc}(\sqrt{\pi n / 2}|\vec{R}+\vec{c}|)}{(n / 2)^{3 / 2}|\vec{R}+\vec{c}|^{3}}\left(1-\frac{6 \eta^{2}}{n|\vec{R}+\vec{c}|^{2}}\right)\right. \\
& \left.+\frac{4 e^{-\pi n|\vec{R}+\vec{c}|^{2} / 2}}{n|\vec{R}+\vec{c}|^{2}}\left(1-\frac{6 \eta^{2}}{n|\vec{R}+\vec{c}|^{2}}-2 \pi \eta^{2}\right)\right] .
\end{aligned}
$$

Finally, the total energy per particle defined in Eq. (4) can be written as

$$
\frac{E}{Q^{2} \sqrt{n}}=\frac{1}{2 \sqrt{2}}(A+B(\eta))+\frac{\mu^{2} n}{Q^{2}} \frac{1}{2^{5 / 2}}(C+D(\eta)) .
$$

Now we define the dimensionless parameter,

$$
\lambda=\frac{\mu^{2} n}{Q^{2}},
$$

which relates the density, the magnetic moment, and the charge of each particle. It is a measure of the relative strength of the magnetic interaction as compared to the Coulomb interaction.
In this case, Eq. (24) takes the form,

$$
\frac{E}{Q^{2} \sqrt{n}}=\frac{1}{2 \sqrt{2}}(A+B(\eta))+\frac{\lambda}{2^{5 / 2}}(C+D(\eta)) .
$$

Because $\lambda$ is associated with the relative strength of the dipole-dipole interaction with respect to the charge-charge interaction, it can be varied experimentally, for example, through an external magnetic field. Notice that the total energy of the system is only a function of $\lambda$ and $\eta$ and therefore the zero temperature $(T=0)$ phase diagram can be represented in $(\lambda, \eta)$ space. The density enters only in the energy (i.e., $\left.E_{0}=Q^{2} \sqrt{n}\right)$ and length $\left(r_{0}=\sqrt{2 / \pi n}\right)$ scales of the problem and in the parameter $\lambda$.

\section{GROUND-STATE CRYSTAL STRUCTURES}

In this section we present the analytical results for the structure of the $T=0$ configurations (ground state).

The ground-state configurations were obtained numerically by comparing the total energy [Eq. (26)] of the nine possible crystalline structures, described in Table I, for both $\vec{c}=0$ (matching) and $\vec{c} \neq 0$ (staggered) cases as a function of $\lambda$ and $\eta$. From all the considered structures the one with the lowest energy is chosen as the ground-state configuration associated with the particular set of parameters $(\lambda, \eta)$.

An example of the total energy as a function of $\eta(\lambda=0.04)$ for the lattices shown in Table I is presented in Fig. 1. Notice that the energy curves cross each other or merge with (or split away from) one another, and these facts are associated with first- and second-order structural phase transitions, which can be observed more clearly in Fig. 1(b). For a first-order structural phase transition, the energy is continuous but the first derivative of the energy with respect to $\eta$ is discontinuous. In this case, the energy curves associated with different structures cross each other. For a second-order transition, the energy and its first derivative are continuous, but the second derivative of the energy with respect to $\eta$ is discontinuous. In this case, the energy curves merge with (or split away from) one another. The transition from the staggered rhombic (SRhomb) to the staggered hexagonal (SH) phase at $\eta \approx 0.65$ is an example of a first-order structural transition, while a second-order structural transition is observed when the system changes from the staggered square (SS) to the SRhomb phase. Notice that such phases differ from each other only in the aspect ratio $a_{2} / a_{1}$ and angle $\theta$ between the primitive vectors. As shown in Fig. 1(c) for $\eta \approx 0.51$ the system starts to change continuously from the SS $\left(\sin \theta=1 ; a_{2} / a_{1}=1\right)$ to the SRhomb phase $(\sin \theta \neq 1)$. 
TABLE I. Lattice parameters of the different crystalline structures. $a$ is the average nearest neighbor distance which is determined by the density and the configuration (see last column). For each case, $\vec{a}_{1}$ and $\vec{a}_{2}$ are the primitive lattice vectors, and $\vec{c}$ is the interlattice displacement vector. $\vec{b}_{1}$ and $\vec{b}_{2}$ are the primitive vectors of the reciprocal lattice. $n$ is the density. The aspect ratio of phases II and VII is $\alpha=a_{2} / a_{1}$. In phases IV and IX, the angle between the lattice vectors $\vec{a}_{1}$ and $\vec{a}_{2}$ is $\theta$.

\begin{tabular}{|c|c|c|c|c|c|c|}
\hline Phases & $\vec{a}_{1} / a$ & $\vec{a}_{2} / a$ & $\vec{c}$ & $\vec{b}_{1} /(2 \pi / a)$ & $\vec{b}_{2} /(2 \pi / a)$ & $n a^{2} / 2$ \\
\hline I. One-component hexagonal (OCH) & $(1,0)$ & $(0, \sqrt{3})$ & $\left(\vec{a}_{1}+\vec{a}_{2}\right) / 2$ & $(1,0)$ & $(0,1 / \sqrt{3})$ & $1 / \sqrt{3}$ \\
\hline II. Staggered rectangular (SRect) & $(1,0)$ & $(0, \alpha)$ & $\left(\vec{a}_{1}+\vec{a}_{2}\right) / 2$ & $(1,0)$ & $(0,1 / \alpha)$ & $1 / \alpha$ \\
\hline III. Staggered square (SS) & $(1,0)$ & $(0,1)$ & $\left(\vec{a}_{1}+\vec{a}_{2}\right) / 2$ & $(1,0)$ & $(0,1)$ & 1 \\
\hline IV. Staggered rhombic (SRhomb) & $(1,0)$ & $(\cos \theta, \sin \theta)$ & $\left(\vec{a}_{1}+\vec{a}_{2}\right) / 2$ & $(1,-\cos \theta / \sin \theta)$ & $(0,1 / \sin \theta)$ & $1 / \sin \theta$ \\
\hline V. Staggered hexagonal (SH) & $(1,0)$ & $(1 / 2, \sqrt{3} / 2)$ & $\left(\vec{a}_{1}+\vec{a}_{2}\right) / 3$ & $(1,-1 / \sqrt{3})$ & $(0,2 / \sqrt{3})$ & $2 / \sqrt{3}$ \\
\hline VI. Matching hexagonal (MH) & $(1,0)$ & $(1 / 2, \sqrt{3} / 2)$ & 0 & $(1,-1 / \sqrt{3})$ & $(0,2 / \sqrt{3})$ & $2 / \sqrt{3}$ \\
\hline VII. Matching rectangular (MRect) & $(1,0)$ & $(0, \alpha)$ & 0 & $(1,0)$ & $\left(0, a_{1} / a_{2}\right)$ & $1 / \alpha$ \\
\hline VIII. Matching square (MS) & $(1,0)$ & $(0,1)$ & 0 & $(1,0)$ & $(0,1)$ & 1 \\
\hline IX. Matching rhombic (MRhomb) & $(1,0)$ & $(\cos \theta, \sin \theta)$ & 0 & $(1,-\cos \theta / \sin \theta)$ & $(0,1 / \sin \theta)$ & $1 / \sin \theta$ \\
\hline
\end{tabular}

We summarize our results in the phase diagram of Fig. 2. The different phases are separated by solid (dotted) lines for first (second)-order structural phase transitions. In the point $(\lambda, \eta)=(0,0)$ the system is found in the one-component hexagonal $(\mathrm{OCH})$ phase, where particles are arranged in a single-layer triangular lattice and the interparticle interaction is only electrostatic. Notice that of the studied nine phases only six are found to be able to become the ground state in a certain area of the $(\lambda, \eta)$ plane. The $\mathrm{OCH}$ phase is also found along the line $\eta=0$, where the magnetic interaction is present $(\lambda \neq 0)$, but in this case the interparticle interaction is only repulsive, since the dipoles are all aligned along the $z$ axis and the interlayer separation is zero. This is the well-known 2D Wigner crystal phase [16]. Along the line $\lambda=0$ the $\mathrm{OCH}$ phase is found only in a very small interval of $\eta$. In fact, already for $\eta=0.006$ the $\mathrm{OCH}$ phase is no longer the ground state. The line $\lambda=0$ corresponds to the case in which the interparticle interaction is only electrostatic. In this case, the system can be found in five energetically favorable staggered configurations (phases I, II, III, IV, and V; see Table I) as a function of $\eta$. The latter results are in complete agreement with those discussed earlier in Ref. [18].

In general, when the magnetic interaction is taken into account $(\lambda \neq 0)$ it is possible to find a configuration in which the dipoles in distinct layers are positioned on top of each other (matching configuration). This phase was absent in Ref. [18] and is a consequence of the attractive magnetic interaction between the particles in different layers. We find that the matching configuration is always reached for a high enough value of $\lambda$ (which is a function of $\eta$ ) through a first-order structural transition (Fig. 2). In this case, the system is always found in the $\mathrm{MH}$ phase, where particles in distinct layers are arranged in a hexagonal lattice and their dipoles are aligned along the $z$ axis. Recently, Xin Lu et al. showed that the MH phase is the ground-state configuration for a 2D classical bilayer system of dipoles oriented perpendicular to the plane of the layers, independently of the interlayer separation and density [19]. They did not include any Coulomb interaction and therefore it corresponds to the case $\lambda \rightarrow \infty$. An interesting point here is that the charging of the dipole particles allows the bilayer system to crystallize in different lattice structures which are not possible when only the magnetic dipole interaction is present.
The critical value of $\lambda$, where the system changes from a staggered (rectangular, square, rhombic, hexagonal) to the MH phase, is a monotonic increasing function of $\eta$. As seen in Fig. 2 we notice two distinct behaviors of $\lambda(\eta)$. Initially, there is a fast increase of $\lambda$ with increasing $\eta$, followed by an almost constant $\lambda(\eta)$. Such a behavior can be qualitatively understood taking into account the range of the Coulomb and magnetic dipole interparticle interaction. An interparticle interaction is defined as short range if it decreases faster than $1 / r^{\alpha}$, where $\alpha$ is the dimensionality of the system [27]. In the opposite case, the interaction is long range. In this sense the Coulomb interaction can be considered as long range and the magnetic dipole interaction as short range. For small $\eta$ the separation between layers is small and the dipole interaction is dominant over the electric interaction. As a consequence, the transition to the $\mathrm{MH}$ phase, which is the ground state for a system with only magnetic dipole interaction, occurs for small $\lambda$. For a large enough separation between the layers the coupling between dipoles in distinct layers (the interlayer interaction) becomes very small. For example, for $\eta=0.8$ the interlayer interaction is only $0.3 \%$ of the total energy. As a consequence, for high enough values of $\eta$ the layers become independent, and it becomes numerically impossible to determine if the $\mathrm{SH}$ or $\mathrm{MH}$ phase is the ground state; for example, for $\eta=2.3(\lambda \approx 0.044)$ the absolute difference in energy between the SH and MH phases is of the order of $10^{-8}$, which is the level of our numerical accuracy. In this case, the total energy is twice the energy of each layer.

A more detailed analysis of the critical $\lambda(\eta)$ which defines the transition from a staggered to the $\mathrm{MH}$ phase identifies a clear crossover between the fast (strong coupling between dipoles in distinct layers) and slow increase of $\lambda(\eta)$. This is shown in Fig. 3, where a $\log \times \log$ plot of the critical $\lambda(\eta)$ curve which separates the staggered phases from the MH phase is presented. As can be seen, there is a power-law increase of $\lambda(\eta)$ for $\eta \lesssim 0.15$ with exponent $\beta \sim 1.92$. Thus for $\eta \lesssim 0.2$ the critical distance between the layers scales as $d \propto(\mu / Q)^{1.04} n^{0.021}$, which indicates a weak dependence on the density and an almost linear dependence on the ratio $\mu / Q$. This scaling behavior can be understood as follows: The interlayer dipole interaction is $\sim \mu^{2} / d^{3}$ while the Coulomb interlayer interaction is $\sim Q^{2} / d$ and therefore we expect the 

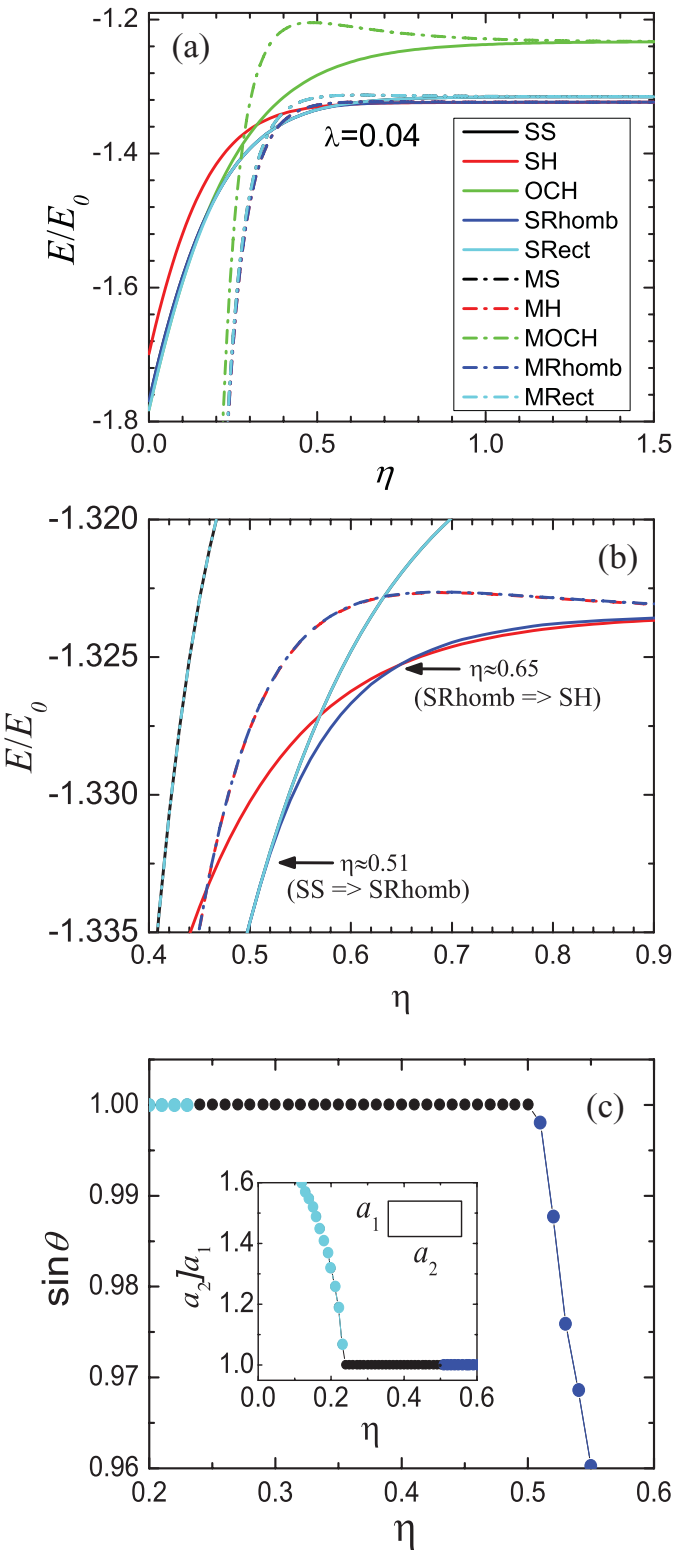

FIG. 1. (Color online) (a) The total energy per particle (in units of $E_{0}=Q^{2} \sqrt{n}$ ) as a function of $\eta$ for the different phases presented in Table I. (b) Detailed view of (a). (c) The sine of the angle between the primitive vectors $\vec{a}_{1}$ and $\vec{a}_{2}$ of the SRhomb phase as a function of $\eta$. The inset in (c) shows how the aspect ratio $a_{2} / a_{1}$ for the SRect phase depends on $\eta$.

staggered to matched transition approximately when $Q^{2} / d \sim$ $\mu^{2} / d^{3}$ and thus $\lambda \sim \eta$.

To conclude, we also present a hatched area in the $(\lambda, \eta)$ phase diagram (Fig. 2). It corresponds to a disordered phase which cannot be obtained from our analytical calculations. The discussions concerning such a phase will be postponed until the next section.

\section{DYNAMICAL PROPERTIES}

Now we turn our discussion to the dynamical properties of the system. Such a study in addition will give us information on

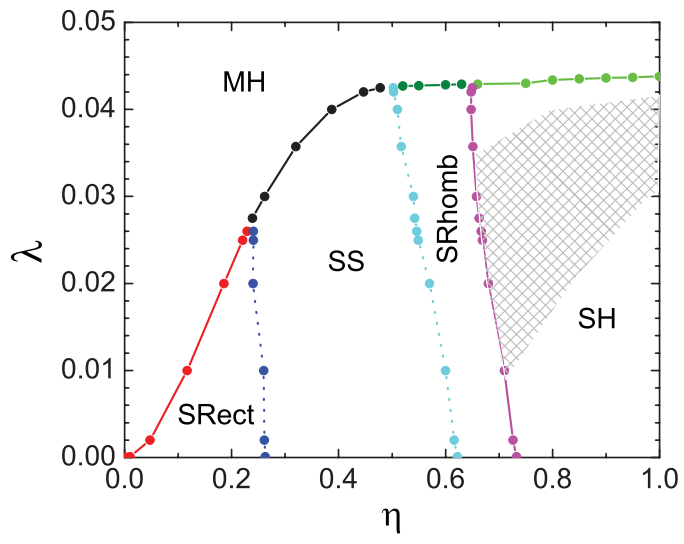

FIG. 2. (Color online) The zero-temperature phase diagram where $\lambda=\mu^{2} n / Q^{2}$ and $\eta=d \sqrt{n / 2}$. First (second)-order structural phase transitions are indicated by solid (dotted) lines. The labels indicating the crystalline phases are given in Table I. The hatched area corresponds to the disordered phase.

the stability of the different phases considered in the previous section. The phonon spectra are calculated within the harmonic approximation. The phonon frequencies for a general lattice are directly obtained from the dynamical matrix through the square root of the eigenvalues. Since we are studying a 2D crystal with two particles per unit cell (one in each layer), the dynamical matrix corresponds to a $4 \times 4$ matrix which can be written as

$$
D=\left(\begin{array}{ll}
D^{A A} & D^{A B} \\
D^{B A} & D^{B B}
\end{array}\right),
$$

where $D^{A A}, D^{A B}, D^{B A}, D^{B B}$ are $2 \times 2$ block matrices which include the intra- and interlayer electric and magnetic interactions. The labels A and B describe the distinct layers, and each block matrix is of the form,

$$
\left[D^{\tau v}(\vec{q})\right]_{\alpha \beta}=\left[D_{e l}^{\tau v}(\vec{q})\right]_{\alpha \beta}+\left[D_{\mathrm{mag}}^{\tau v}(\vec{q})\right]_{\alpha \beta},
$$

where $\tau, v=A, B ; \alpha, \beta=x, y$. Following the procedure described in Ref. [18] and by using Eqs. (12a), (12b), (19a),

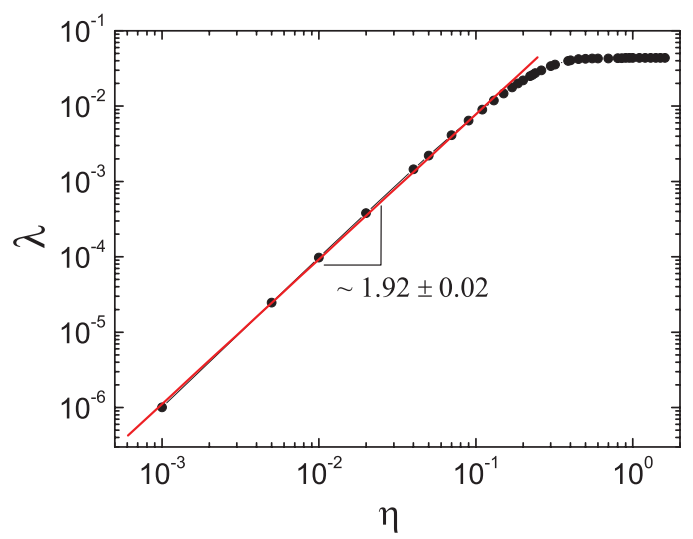

FIG. 3. (Color online) The $\log \times \log$ plot of the critical $\lambda(\eta)$ curve which separates the staggered phases from the $\mathrm{MH}$ phase taken from Fig. 2. 
and (19b), the different terms present in Eq. (28) are given by

$$
\begin{aligned}
& {\left[D_{e l}^{A A}(\vec{q})\right]_{\alpha \beta}=\frac{1}{m}\left\{\left[S_{e l}^{A A}(0)\right]_{\alpha \beta}+\left[S_{e l}^{A B}(0)\right]_{\alpha \beta}-\left[S_{e l}^{A A}(\vec{q})\right]_{\alpha \beta}\right\},} \\
& {\left[D_{e l}^{A B}(\vec{q})\right]_{\alpha \beta}=\frac{1}{m}\left\{-\left[S_{e l}^{A B}(\vec{q})\right]_{\alpha \beta}\right\},} \\
& {\left[D_{\mathrm{mag}}^{A A}(\vec{q})\right]_{\alpha \beta}=\frac{1}{m}\left\{\left[S_{\mathrm{mag}}^{A A}(0)\right]_{\alpha \beta}+\left[S_{\mathrm{mag}}^{A B}(0)\right]_{\alpha \beta}-\left[S_{\mathrm{mag}}^{A A}(\vec{q})\right]_{\alpha \beta}\right\},} \\
& {\left[D_{\mathrm{mag}}^{A B}(\vec{q})\right]_{\alpha \beta}=\frac{1}{m}\left\{-\left[S_{\mathrm{mag}}^{A B}(\vec{q})\right]_{\alpha \beta}\right\},}
\end{aligned}
$$

where $m$ is the mass of each particle and

$$
\left[S_{e l}^{A A}(\vec{q})\right]_{\alpha \beta}=-Q^{2} \lim _{r \rightarrow 0} \partial_{\alpha} \partial_{\beta} T_{0}(\vec{r}, \vec{q})=-Q^{2} \sqrt{n_{s}}[E(\vec{q})]_{\alpha \beta},
$$

$\left[S_{e l}^{A B}(\vec{q})\right]_{\alpha \beta}=-Q^{2} \lim _{r \rightarrow 0} \partial_{\alpha} \partial_{\beta} T_{I}(\vec{r}, \vec{q})=-Q^{2} \sqrt{n_{s}}[F(\vec{q}, \eta)]_{\alpha \beta}$,

$$
\left[S_{\mathrm{mag}}^{A A}(\vec{q})\right]_{\alpha \beta}=-\mu^{2} \lim _{r \rightarrow 0} \partial_{\alpha} \partial_{\beta} \psi_{0}(\vec{r}, \vec{q})=-\mu^{2} \sqrt{n_{s}}[G(\vec{q})]_{\alpha \beta},
$$

$\left[S_{\mathrm{mag}}^{A B}(\vec{q})\right]_{\alpha \beta}=-\mu^{2} \lim _{r \rightarrow 0} \partial_{\alpha} \partial_{\beta} \psi_{I}(\vec{r}, \vec{q})=-\mu^{2} \sqrt{n_{s}}[H(\vec{q}, \eta)]_{\alpha \beta}$.

The auxiliary functions $[E(\vec{q})]_{\alpha \beta},[F(\vec{q}, \eta)]_{\alpha \beta},[G(\vec{q})]_{\alpha \beta}$, and $[H(\vec{q}, \eta)]_{\alpha \beta}$ are given by

$$
\begin{gathered}
{[E(\vec{q})]_{\alpha \beta}=-\sum_{\vec{G}}(\vec{q}+\vec{G})_{\alpha}(\vec{q}+\vec{G})_{\beta} \Phi\left(\frac{|\vec{q}+\vec{G}|^{2}}{4 \pi n_{s}}\right)+\lim _{r \rightarrow 0} \sum_{\vec{R} \neq \overrightarrow{0}} e^{-i \vec{q} \cdot \vec{R}} \partial_{\alpha} \partial_{\beta} \Phi\left(\pi n_{s}|\vec{r}-\vec{R}|^{2}\right)+\delta_{\alpha \beta} \frac{4}{3} \pi n_{s},} \\
{[F(\vec{q}, \eta)]_{\alpha \beta}=-\sum_{\vec{G}}(\vec{q}+\vec{G})_{\alpha}(\vec{q}+\vec{G})_{\beta} e^{-i \vec{G} \cdot \vec{c}} \Psi\left(\frac{|\vec{q}+\vec{G}|^{2}}{4 \pi n_{s}}, \pi \eta^{2}\right)+\lim _{r \rightarrow 0} \sum_{\vec{R}} e^{-i \vec{q} \cdot(\vec{R}-\vec{c})} \partial_{\alpha} \partial_{\beta} \Phi\left(\pi\left[n_{s}|\vec{r}-\vec{R}+\vec{c}|^{2}+\eta^{2}\right]\right),} \\
{[G(\vec{q})]_{\alpha \beta}=-\pi n_{s} \sum_{\vec{G}}(\vec{q}+\vec{G})_{\alpha}(\vec{q}+\vec{G})_{\beta} \Upsilon\left(\frac{|\vec{q}+\vec{G}|}{2 \varepsilon}, 0\right)+\lim _{r \rightarrow 0} \sum_{\vec{R} \neq \overrightarrow{0}} e^{-i \vec{q} \cdot \vec{R}} \partial_{\alpha} \partial_{\beta} \Omega_{1}(|\vec{r}+\vec{R}|)+\delta_{\alpha \beta} \frac{8 \varepsilon^{5}}{5 \sqrt{\pi}}} \\
{[H(\vec{q}, \eta)]_{\alpha \beta}=-\pi n_{s} \sum_{\vec{G}}(\vec{q}+\vec{G})_{\alpha}(\vec{q}+\vec{G})_{\beta} e^{i \vec{G} \cdot \vec{c}} \Upsilon\left(\frac{|\vec{q}+\vec{G}|}{2 \varepsilon}, \varepsilon d\right)+\lim _{r \rightarrow 0} \sum_{\vec{R}} e^{-i \vec{q} \cdot(\vec{R}+\vec{c})} \partial_{\alpha} \partial_{\beta} \Omega_{2}(|\vec{r}+\vec{R}+\vec{c}|),}
\end{gathered}
$$

and the functions $\Upsilon(x, y), \Omega_{1}(x)$, and $\Omega_{2}(x)$ which appear in Eqs. (31c) and (31d) are

$$
\begin{aligned}
\Upsilon(x, y) & =\frac{4 \varepsilon}{\sqrt{\pi}} e^{-x^{2}-y^{2}}+\sum_{ \pm}(-2) \varepsilon x e^{ \pm 2 x y} \operatorname{erfc}(x \pm y), \\
\Omega_{1}(x) & =\frac{\operatorname{erfc}(\varepsilon x)}{x^{3}}+\frac{2 \varepsilon e^{-\varepsilon^{2} x^{2}}}{\sqrt{\pi} x^{2}}, \\
\Omega_{2}(x) & =\frac{\operatorname{erfc}(\varepsilon x)}{x^{3}}+\frac{2 \varepsilon e^{-\varepsilon^{2} x^{2}}}{\sqrt{\pi} x^{2}}-3 d^{2}\left[\frac{\operatorname{erfc}(\varepsilon x)}{x^{5}}+\frac{2 \varepsilon\left(3+2 \varepsilon^{2} x^{2}\right) e^{-\varepsilon^{2} x^{2}}}{3 \sqrt{\pi} x^{4}}\right] .
\end{aligned}
$$

By using the relations $\varepsilon=1 / r_{0}=\sqrt{\pi n_{s}}, n_{s}=n / 2$, and $\lambda=\mu^{2} n / Q^{2}$ the terms of the matrix given in Eq. (27) become

$$
\begin{aligned}
& {\left[D^{A A}(\vec{q})\right]_{\alpha \beta}=\frac{-Q^{2} n^{3 / 2}}{m}\left[\frac{1}{2^{3 / 2} n_{s}}\left\{[E(0)]_{\alpha \beta}+[F(0, \eta)]_{\alpha \beta}-[E(\vec{q})]_{\alpha \beta}\right\}+\frac{\lambda}{\left(2 n_{s}\right)^{5 / 2}}\left\{[G(0)]_{\alpha \beta}+[H(0, \eta)]_{\alpha \beta}-[G(\vec{q})]_{\alpha \beta}\right\}\right],} \\
& {\left[D^{A B}(\vec{q})\right]_{\alpha \beta}=\frac{Q^{2} n^{3 / 2}}{m}\left[\frac{1}{2^{3 / 2} n_{s}}[F(\vec{q}, \eta)]_{\alpha \beta}+\frac{\lambda}{\left(2 n_{s}\right)^{5 / 2}}[H(\vec{q}, \eta)]_{\alpha \beta}\right] .}
\end{aligned}
$$

The two layers of particles are equivalent. In this case, $D^{A A}=D^{B B}, D^{A B}=\left[D^{B A}\right]^{\dagger}$, and the dynamical matrix $D$ may now be calculated as a function of $\lambda, \vec{q}$, and $\eta$. In general, the dynamical matrix is complex Hermitian. Therefore, it is possible to apply a unitary transformation in order to generate a real and symmetric matrix. Such a transformation is given by the matrix,

$$
U=\frac{1}{\sqrt{2}}\left(\begin{array}{cc}
I_{2} & i I_{2} \\
i I_{2} & I_{2}
\end{array}\right)
$$

where $I_{2}$ is the $2 \times 2$ identity matrix, and

$$
\bar{D}=U D U^{-1}=\left(\begin{array}{cc}
D^{A A}+\operatorname{Im} D^{A B} & \operatorname{Re} D^{A B} \\
\operatorname{Re} D^{A B} & D^{A A}-\operatorname{Im} D^{A B}
\end{array}\right),
$$

where $\operatorname{Re} D^{A B}$ and $\operatorname{Im} D^{A B}$ are the real and imaginary parts of $D^{A B}$, respectively. Since a unitary transformation does not change the eigenvalues, we may consider now the real and symmetric matrix $\bar{D}$ in order to obtain the eigenvalues and the phonon frequencies. For each $(\lambda, \eta)$, which specify a given structure in the phase diagram shown in Fig. 2, we vary the 
wave vector $\vec{q}$ along a given symmetry direction of the first Brillouin zone of the corresponding phases. For each value of $\vec{q}$ we generate a $4 \times 4$ matrix which gives us four eigenvalues $\omega_{j}^{2}(\vec{q}) / \omega_{0}^{2}$, with $j=1, \ldots, 4$ and $\omega_{0}^{2}=Q^{2} n^{3 / 2} / m$, and for each eigenvalue a corresponding eigenvector $\vec{e}(\vec{q}, j)$ which indicates the direction of the phonon oscillation.

In our analysis of the dispersion relation we will present only frequencies which are real positive [i.e., $\left.\omega_{j}^{2}(\vec{q}) / \omega_{0}^{2} \geqslant 0\right]$. For $\omega^{2}<0$ the frequencies are imaginary, which means that the amplitudes of particle oscillation become an exponentially increasing function of time. In this case the crystalline structure is unstable and will not exist. As commented earlier, in all phases studied in the previous section there are two particles per unit cell, one in each layer. As a consequence, there are two acoustic and optical modes which are associated with the inphase and out-of-phase vibrations of particles in the unit cell, respectively. The acoustic branch is characterized by $\omega(\vec{q}) \rightarrow$ 0 for $\vec{q} \rightarrow 0$, while in the optical branch $\omega(\vec{q}) \rightarrow$ constant in the limit $\vec{q} \rightarrow 0$. Besides, the acoustic and optical branches may also be defined as longitudinal, $\vec{e} \| \vec{q}$, and transverse mode [28], $\vec{e} \perp \vec{q}$. Due to the extended parameter space, we present here only some examples which illustrate the general behavior of the phonon spectrum.

In general, we find qualitative distinct behaviors for the normal mode spectra for the staggered phases and for the matching hexagonal phase. With exception of the SH phase, for a given staggered phase the phonon spectrum is almost independent of $\lambda$, but it depends strongly on the parameter $\eta$. In addition, for a given high-symmetry direction of the reciprocal space we found a monotonic increasing (or decreasing) behavior of the phonon frequencies as a function of $\eta$. As an example, the phonon frequencies for the SS phase along the high-symmetry directions of the reciprocal space are presented in Fig. 4 for $\lambda=0.002$ and $\lambda=0.029$ and different $\eta$. The high-symmetry points in the first Brillouin zone are indicated in the insets. Notice that for both values of $\lambda$, which are one order of magnitude distinct, the same qualitative behavior is found for the phonon frequencies as a function of the wave vector. The phonons soften with increasing $\eta$ along the $\Gamma \mathrm{X}$ direction. Along the XM direction, the normal mode frequencies are degenerate for any value of $\eta$. The lowest normal mode frequencies cross at a specific $q$ value which is independent of $\eta$. Notice that in the $\Gamma \mathrm{M}$ direction the lowest energy phonons soften with decreasing $\eta$ which is the opposite behavior found along the $\Gamma \mathrm{X}$ direction.

For the MH phase we present in Fig. 5 the phonon spectrum for $\lambda=0.046$. In this case, the bilayer system is found in the MH phase for any value of $\eta$. Again the high-symmetry points in the reciprocal space are labeled in the inset. For a fixed density $n$, the parameter $\eta=d \sqrt{n / 2}$ is directly related to the separation between the layers. For $\eta=0.1$ a large gap between the acoustic and optical modes is observed. The later ones, which describe the out-of-phase vibrations of particles in distinct layers, are two orders of magnitude larger than the acoustic ones. Such a behavior is due to the strong dipolar magnetic interaction for small $\eta$. Notice that the dipole interaction $\left(\propto 1 / r^{3}\right)$ is dominant over the Coulomb interaction $(\propto 1 / r)$ for short distances $r$. The acoustic modes, which describe the in-phase oscillation of particles in the unit cell (distinct layers), are almost not
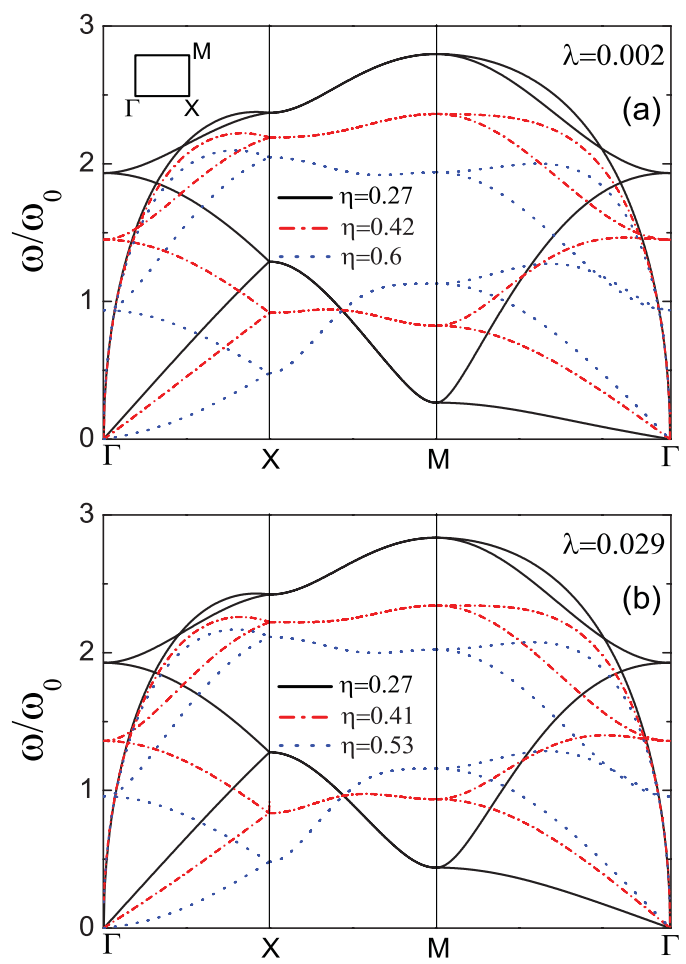

FIG. 4. (Color online) The phonon spectrum for the staggered square phase for different values of $\eta$ and for (a) $\lambda=0.002$ and (b) $\lambda=0.029$. The high-symmetry directions of the reciprocal space are presented in the insets. The frequency is in units of $\omega_{0}=Q n^{3 / 4} / m^{1 / 2}$.

affected by the dipole coupling. In addition, the width of the optical band becomes extremely narrow. For large separation of the layers $(\eta=0.8)$ all mode frequencies have the same order of magnitude (Fig. 5), indicating a weaker coupling between dipoles in distinct layers. This is in agreement with the comment made in the previous section that, for $\eta=0.8$ the interlayer interaction is only $0.3 \%$ of the total energy. The gap between the acoustic and optical modes is observed for $\eta \lesssim 0.44$. For $\eta \gg 1$ the acoustic and optical modes (transverse and longitudinal) become degenerate since

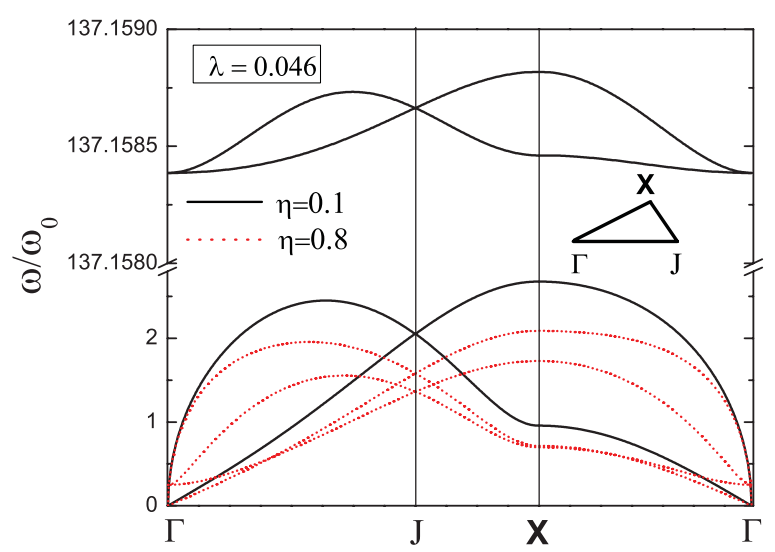

FIG. 5. (Color online) The phonon spectrum for the MH phase for different values of $\eta$ and fixed $\lambda$. The high-symmetry directions of the reciprocal space are presented in the inset. 


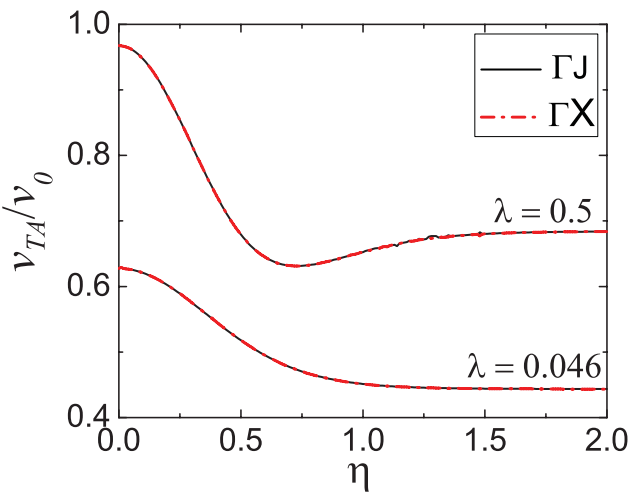

FIG. 6. (Color online) The sound velocity (in units of $v_{0}=$ $\omega_{0} / \sqrt{n}$ ) of the TA mode as a function of $\eta$ for $\lambda=0.046$ and $\lambda=0.5$.

the coupling between the layers becomes very small, and the system behaves as two independent single-layer systems.

As shown previously for the SS phase, we also find a monotonic behavior of the phonon spectrum of the $\mathrm{MH}$ phase for $\lambda=0.046$ (i.e., there is a softening of the phonon frequencies with increasing $\eta$ for all the high-symmetry directions of the reciprocal space). Such a behavior is interesting, since in the bilayer system with only dipole interaction, a nonmonotonic behavior of the phonon spectrum was observed as a function of $\eta$ [19]. Such a behavior was explained in Ref. [19] as being linked to the competitive character of the dipole-dipole interaction. In that case, the nonmonotonic behavior of the phonon spectrum also revealed a nonmonotonic dependence of the sound velocity on $\eta$. In addition, for $\eta \rightarrow 0$ the sound velocity is a factor $\sqrt{2}$ larger than the value obtained for $\eta \gg 1$, showing that for small separations the bilayer system of dipoles behaves as a crystal of particles with twice larger dipole moment and mass. In the present bilayer system of charged dipole particles, we found that such a nonmonotonic behavior for the phonon spectrum depends on the parameter $\lambda$ as shown in Fig. 6. For $\lambda=0.046$ the sound velocity is a monotonic function of $\eta$, but, for example, for $\lambda=0.5$ it is nonmonotonic exhibiting a minimum for $\eta=0.73$. We found that the nonmonotonic versus monotonic behavior of the sound velocity is associated with a change in the attractive or repulsive character of the total energy (i.e., it is attractive when $v_{T A}$ is nonmonotonic). This is an interesting feature, since in electrically steric colloidal systems the charge adsorbed on the colloidal particles can be controlled, for example, by changing the PH of the solution [25]. In addition, since the melting temperature can in principle be calculated from the normal mode frequencies, the nonmonotonic behavior of the phonon spectrum should play an important role in the behavior of the melting temperature, which should become noticeable when varying $\lambda$.

As shown in Fig. 2 , for $\eta \gtrsim 0.732(\lambda \gtrsim 0.35)$ the bilayer system can be found in either the SH or MH phases, depending on $\lambda$. Note that $\lambda$ can be varied either through $Q$ or $\mu$. Now we study how the phonon spectrum changes as a function of $\lambda$ in the case where the hexagonal phase is found as the ground state in each layer. This is shown in Fig. 7 where the phonon spectra for different values of $\lambda$ are presented for $\eta=0.8$. As shown in Fig. 2, a structural first-order phase transition from

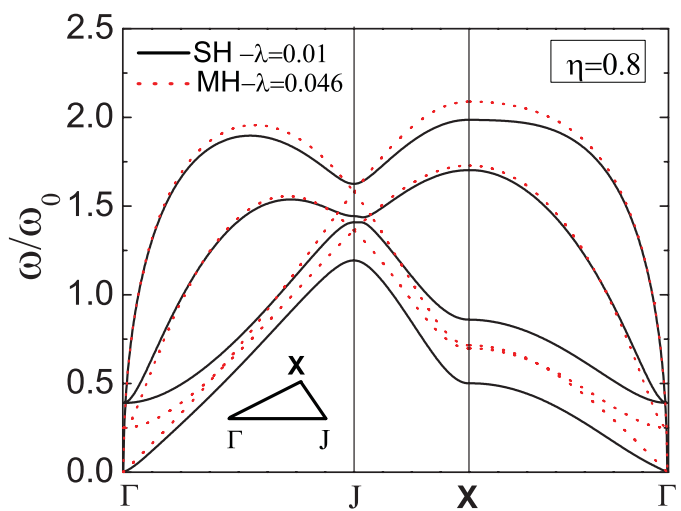

FIG. 7. (Color online) The phonon spectrum for the $\mathrm{SH}$ and $\mathrm{MH}$ phases for different $\lambda$ and fixed $\eta=0.8$. The high-symmetry direction of the reciprocal space are presented in the inset.

the SH to the MH phase is observed with increasing $\lambda$. For $\lambda \gtrsim 0.0436$ the $\mathrm{MH}$ phase appears as the ground state. It is interesting to notice that for $\eta=0.8(\lambda=0.046)$ the layers are sufficiently far apart in order that the optical and acoustical frequencies are of the same order of magnitude, indicating a weak dipole-dipole coupling between layers. However, the magnetic interaction still plays an important role since the MH phase is found as the ground state. The optical modes are softened and the acoustical modes are hardened when the system changes from the $\mathrm{SH}$ to the $\mathrm{MH}$ phase.

Now we study the interval of stability of the different phases (deduced from the phonon spectrum) and compare it with the position of the phase boundary (obtained from the minimum energy criterion).

As expected, not only the phase boundary but also the stability of the MH phase is enhanced with increasing $\lambda$ in the sense that the interval of $\eta$ increases with increasing $\lambda$. This is shown in Fig. 8 for $\lambda=0.01$ and 0.03 . Notice that the stability interval of the MH phase is larger than the phase boundaries in both cases, indicating that the MH phase is metastable beyond the phase boundary. In such a case the structural phase transition is first order. The interval of stability of the staggered phases with increasing $\lambda$ depends on the crystalline structure. For the $\mathrm{OCH}$, which is not found as a ground state for $\lambda \neq 0$, the interval of stability decreases with increasing $\lambda$ (Fig. 8). For the SS phase and $\lambda=0.03$ the interval of stability of the SS phase $(0.238 \lesssim \eta \lesssim 0.534)$ becomes larger than its phase boundary $(0.262 \lesssim \eta \lesssim 0.534)$ and a first-order transition separates the SS and MH phases. When the SS phase is bordered by the SRect and SRhomb phases $(\lambda \lesssim 0.027)$ its phase boundary and interval of stability coincide and these phases are separated by a second-order structural (continuous) transition, characterized by the softening of one of the phonon mode frequencies. The SRect phase, which is suppressed as a ground-state configuration for $\lambda \gtrsim 0.027$, is still stable as shown, for example, for $\lambda=0.03$.

An interesting feature of the present system, found for $\lambda \neq 0$, is that more than two phases can be stable for some interval of $\eta$. For example, for $\eta=0.24(\lambda=0.03)$ the $\mathrm{MH}$, $\mathrm{SS}$, and $\mathrm{OCH}$ phases are all stable. In contrast, for the pure Coulomb [18] and magnetic [19] systems a maximum number of two phases were found to be stable in a given 

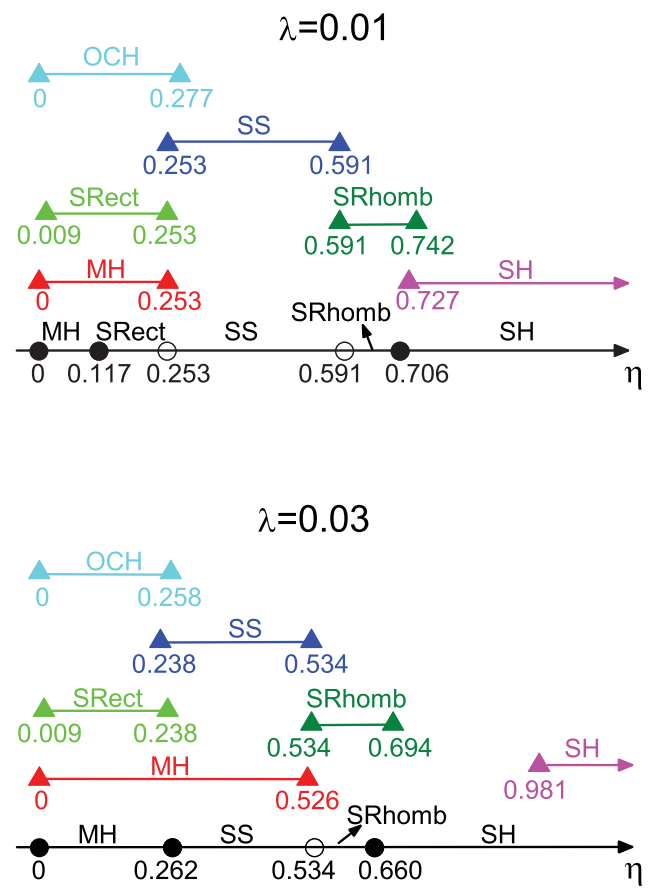

FIG. 8. (Color online) The phase boundaries (circles) and the range of stability (colored triangles) for the different phases as a function of $\eta$ for two values of $\lambda$. Solid (open) circles refer to first (second)-order structural phase transitions.

interval of $\eta$. The presence of many stable phases might have important consequences for the melting temperature. In this case, structural transitions between such phases should, in principle, be possible before the system melts.

From Fig. 8 we notice that in the large $\eta$ region (hatched) in the $(\lambda, \eta)$ phase diagram (Fig. 2) there is a discrepancy between the found lowest energy structure and its stability. For $\lambda=0.01$ we found that the $\mathrm{SH}$ configuration has the lowest energy for $\eta>0.706$ while it is only stable for $\eta>0.727$. For $\eta<0.727$ the frequency of the transverse acoustic mode of the SH phase becomes imaginary along the $\Gamma X$ and $\Gamma J$ directions. Imaginary frequency is also found for the other phases presented in Table I. From this observation we are forced to conclude that in the region $0.706<\eta<0.727$ none of the nine crystal structures can be the ground state. This discrepancy is even more pronounced for $\lambda=0.03$ where the $\mathrm{SH}$ phase was found to be unstable in the range $0.660<\eta<$ 0.981 where (from the analytical calculations) it was initially predicted to be the ground state (Fig. 2). An important lesson to be learned from this stability analysis is that one has to be very careful to rely only on the most plausible crystal structures in combination with an energy minimization when deciding which phase is the ground state. In order to find the true ground state in this area of the phase diagram we resorted to a pure numerical approach.

We used Monte Carlo (MC) numerical simulations in order to find the stable ground-state configuration. As an example we took $\lambda=0.03$ and $\eta=0.8$ and we notice from the inset of Fig. 9(a) that the obtained ground-state configuration is similar to the $\mathrm{SH}$ phase [inset Fig. 9(c)], but the 2D displacement of one layer with respect to the other one

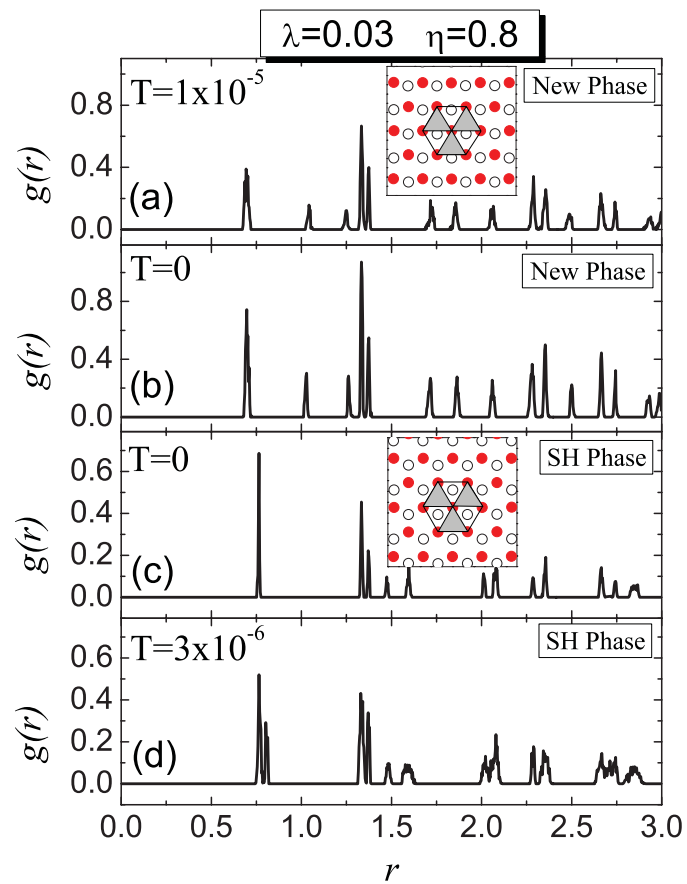

FIG. 9. (Color online) The radial distribution function as calculated from our MC simulations for the new phase and the $\mathrm{SH}$ phase for two different temperatures. For the new phase, (a) $T=1 \times 10^{-5}$ and (b) $T=0$. For the SH phase: (c) $T=0$ and (d) $T=3 \times 10^{-6}$. The configuration of the new phase (energy $E=-1.340575$ ) is presented as an inset in (a), while the configuration of the SH phase (energy $E=-1.340534)$ is presented as an inset in (c). The $T \neq 0$ results in (a) and (d) were obtained by applying MC simulations starting with the new phase and the SH phase at $T=0$, respectively. Solid and open circles represent particles in distinct layers.

is different [i.e., $\vec{c} \neq\left(\vec{a}_{1}+\vec{a}_{2}\right) / 3$ ]. The energy of this new phase is slightly lower (i.e., the difference with the $\mathrm{SH}$ phase is $\Delta E \approx 10^{-5}$ ).

To test numerically the stability of the new configuration [inset of Fig. 9(a)] we compare the $T=0$ and $T \neq 0$ pair distribution functions $g(r)$ calculated from the $\mathrm{MC}$ simulations. Remark that $g(r)$ contains both the interlayer $\left[g_{12}(r)\right]$ and the intralayer $\left[g_{i i}(r)\right]$ radial distribution, where the latter takes into account only the in-plane component. As can be observed from Fig. 9(a) the $g(r)$ function remains almost unaltered when we increase $T$ slightly, indicating the thermal stability of the phase. That the $\mathrm{SH}$ phase is indeed unstable we tested by using our MC simulations and let the program run for $T \neq 0$. Notice that the $g(r)$ for $T=0$ and the one for very low temperature $T=3 \times 10^{-6}$ are different [Figs. 9(c) and 9(d)]. There is a clear disordering of the lattice which indicates that very small thermal fluctuations destroy already the $\mathrm{SH}$ phase and consequently the $\mathrm{SH}$ phase is indeed unstable.

From the inset of Fig. 9(a) it appears that in the new phase both lattices are slightly shifted with respect to each other. This is reflected in $g(r)$ where the first peak now appears at a slightly smaller $r$ value and there is a second peak for $r \approx 1$ which is not present in the SH phase. These two peaks reflect only the short-range interlayer ordering. In fact, a more careful analysis reveals that the new phase does not consist of a 


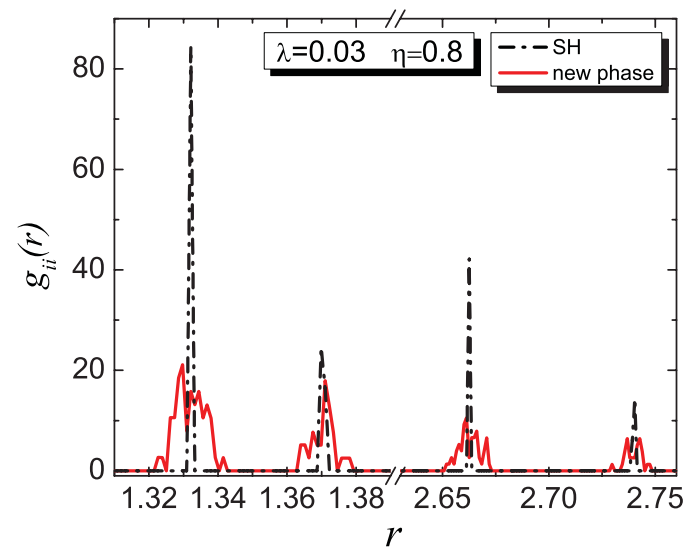

FIG. 10. (Color online) The radial distribution function as calculated from our MC simulations $(T=0)$ taking into account only one layer of the SH phase (dash-dotted black curve) and one layer of the new phase (solid red curve).

perfect hexagonal configuration in each layer. This can be seen in Fig. 10 where a comparison between the pair distribution functions $g_{i i}(r)$ calculated in each layer of the new phase and the one calculated in each layer of the $\mathrm{SH}$ phase is presented in two narrow ranges of $r$. The difference observed in the $g_{i i}(r)$ functions between both phases indicates that the new phase does not consist of a perfect hexagonal lattice in each layer. Notice that for each peak of the SH phase, there appear many peaks (or a broadening of the hexagonal lattice peak) of the new phase around it, which indicates that the lattice is distorted (or strained).

\section{CONCLUSION}

We studied a 2D classical bilayer system of charged magnetic dipoles. The phase diagram at $T=0$, as well as the phonon spectra were obtained through minimization of the energy and within the harmonic approximation, respectively. We obtained a very rich phase diagram at $T=0$ with six different crystalline structures, being five staggered phases (OCH, SS, SRect, SRhomb, SH), which were previously found as the ground-state configuration when no magnetic interaction is present [18], and an MH phase, which was obtained as the only ground-state configuration for the bilayer system of dipoles aligned perpendicularly to the layers [19]. Notice that the presence of both Coulomb and magnetic interaction allows the appearance of phases which were not found in the pure Coulomb (MH phase) and magnetic systems (staggered phases). In the latter, the charges on the dipole particles allow the bilayer system to crystallize in different lattice structures which are not possible when only the magnetic dipole interaction is present (e.g., the SH and SRhomb phases appear not stable for any interval of $\eta$ ). The phase diagram was obtained as a function of the separation between the layers $(\eta)$, and a parameter $(\lambda)$ which is associated with the relative strength of the magnetic and Coulomb interaction between the particles. We found that the staggered phase boundaries depend on $\lambda$. For example, the SRect phase is no longer the ground state for $\lambda \gtrsim 0.027$.
The phonon spectrum of the different phases given in the $(\lambda, \eta)$ phase diagram was obtained. With the exception of the $\mathrm{SH}$ phase, we found that for a given staggered phase the phonon spectrum has the same qualitative behavior for different $\lambda$, but depends sensitively on the separation between the layers $\eta$. For the MH phase, there is a strong dependence of the phonon spectrum on $\eta$. For small $\eta$ the optical frequencies become very large due to the strong coupling between dipoles in the distinct layers. In addition the optical band becomes very narrow. In addition, a nonmonotonic behavior of the phonon spectrum as a function of $\lambda$ was found for the MH phase, which is related to the competition between the dipole and the Coulomb interaction [19]. We found that the nonmonotonic behavior of the phonon spectrum is associated with a change from attractive to repulsive character in the total energy. Notice that in electrically steric colloidal systems the charge adsorbed on the colloidal particles can be controlled, for example, by changing the $\mathrm{pH}$ of the solution [25]. In this case, for a large enough separation between the layers, where only the $\mathrm{MH}$ and $\mathrm{SH}$ phases are found as ground state, it is possible to tune the configuration between staggered and matching by changing, for example, the charge on the particles (fixed $\mu$ ) and, consequently, $\lambda$. Alternatively, we may change the magnetic field strength in order to tune the value of $\lambda$. In addition, since the melting temperature can, in principle, be calculated from the normal mode frequencies (at least within the harmonic approximation), the nonmonotonic behavior of the phonon spectrum might play an important role when determining the melting temperature for different $\lambda$.

The stability of the phases obtained from the phonon spectrum were compared with the phase boundaries for different values of $(\lambda, \eta)$. In particular, the MH phase is enhanced with increasing $\lambda$, in the sense that a larger phase boundary and interval of stability is observed. The SRect phase, which is no longer observed as a ground-state configuration for $\lambda \gtrsim 0.027$ still appears as a metastable configuration. As an important finding, the presence of both electric and magnetic interaction stabilizes up to three phases in some $\eta$ interval of a given ground-state configuration, and this fact should have profound implications on the melting temperature, since structural transitions may take place for temperatures $T \neq 0$.

We found a region in the $(\lambda, \eta)$ phase diagram where the SH phase has the lowest energy among the considered nine crystal structures while from the phonon spectrum it appears to be unstable. Monte Carlo simulations were used to determine the ordered structure in this region, and we found that the lowest energy configuration corresponds to a distorted hexagonal lattice structure, where the lattice positions are slightly disordered.

\section{ACKNOWLEDGMENTS}

This work was supported by the Brazilian agencies CNPq, CAPES, and FUNCAP (PRONEX grant), the Flemish Science Foundation (FWO-Vl), the bilateral program between Flanders and Brazil, and the CNPq-FWO collaborating project. The authors are grateful to Prof. G. Goldoni for some technical clarifications concerning Ref. [18]. 
[1] E. P. Wigner, Phys. Rev. 46, 1002 (1934).

[2] C. C. Grimes and G. Adams, Phys. Rev. Lett. 42, 795 (1979).

[3] D. W. Wang, M. D. Lukin, and E. Demler, Phys. Rev. Lett. 97, 180413 (2006)

[4] D. W. Wang, Phys. Rev. Lett. 98, 060403 (2007).

[5] H. P. Buchler, E. Demler, M. D. Lukin, A. Micheli, N. V. Prokof'ev, G. Pupillo, and P. Zoller, Phys. Rev. Lett. 98, 060404 (2007).

[6] M. Golosovsky, Y. Saado, and D. Davidov, Phys. Rev. E 65, 061405 (2002).

[7] K. Zahn, R. Lenke, and G. Maret, Phys. Rev. Lett. 82, 2721 (1999); K. Zahn and G. Maret, ibid. 85, 3656 (2000).

[8] S. Neser, C. Bechinger, P. Leiderer, and T. Palberg, Phys. Rev. Lett. 79, 2348 (1997).

[9] A. D. Law, D. M. Buzza, and T. S. Horozov, Phys. Rev. Lett. 106, 128302 (2011).

[10] A. D. Law, T. S. Horozov, and D. M. Buzza, Soft Matter 7, 8923 (2011).

[11] J. Fornleitner, G. Kahl, and C. N. Likos, Phys. Rev. E 81, 060401 (2010).

[12] J. H. Chu and Lin I, Phys. Rev. Lett. 72, 4009 (1994).

[13] G. Coupier, C. Guthmann, Y. Noat, and M. Saint Jean, Phys. Rev. E 71, 046105 (2005).

[14] Peter Schall, Itai Cohen, David A. Weitz, and Frans Spaepen, Nature (London) 440, 319 (2006).
[15] W. P. Ferreira, A. Matulis, G. A. Farias, and F. M. Peeters, Phys. Rev. E 67, 046601 (2003).

[16] L. Bonsall and A. A. Maradudin, Phys. Rev. B 15, 1959 (1977); F. M. Peeters, ibid. 30, 159 (1984).

[17] D. S. Fisher, Phys. Rev. B 26, 5009 (1982).

[18] G. Goldoni and F. M. Peeters, Phys. Rev. B 53, 4591 (1996).

[19] Xin Lu, Chang-Qin Wu, Andrea Micheli, and Guido Pupillo, Phys. Rev. B 78, 024108 (2008).

[20] J. Dobnikar, J. Fornleitner, and G. Kahl, J. Phys.: Condens. Matter 20, 494220 (2008).

[21] Julia Fornleitner, Federica Lo Verso, Gerhard Kahl, and Christos N. Likos, Soft Matter 4, 480 (2008).

[22] L. Assoud, F. Ebert, P. Keim, R. Messina, G. Maret, and H. Löwen, J. Phys.: Condens. Matter 21, 464114 (2009).

[23] Angang Dong, Xingchen Ye, Jun Chen, and Christopher B. Murray, Nano Lett. 11, 1804 (2011).

[24] A. Delattre, S. Pouget, J. F. Jacuot, Y. Samson, and P. Reiss, Small 6, 932 (2010).

[25] A. F. C. Campos, F. A. Tourinho, G. J. da Silva, M. C. F. L. Lara, and J. Depeyrot, Eur. Phys. J. E 6, 29 (2001).

[26] F. M. Peeters and X. G. Wu, Phys. Rev. A 35, 3109 (1987).

[27] M. P. Allen and D. J. Tildesley, Computer Simulation of Liquids (Clarendon Press, Oxford, 1992).

[28] G. P. Srivastava, The Physics of Phonons (Adam Hilger, Bristol, 1990). 